\title{
Cerambycinae (Coleoptera, Cerambycidae) em Mato Grosso, Brasil*
}

\author{
Marliton Rocha Barreto ${ }^{1,3},{\text { Ricardo } \text { Machiner }^{2} \text { \& Eder Cristian Smiderle }}^{2}$ \\ ${ }^{1}$ Núcleo de Estudos da Biodiversidade da Amazônia Mato-grossense-NEBAM, Instituto de Ciências \\ Naturais, Humanas e Sociais - ICNHS, Universidade Federal de Mato Grosso - UFMT, \\ Av. Alexandre Ferronato, 1200, CEP 78557-267, Sinop, MT, Brasil. http://www.ufmt.br \\ ${ }^{2}$ Instituto de Ciências Agrárias e Ambientais - ICAA, Universidade Federal de Mato Grosso - UFMT, \\ Av. Alexandre Ferronato, 1200, CEP 78557-267, Sinop, MT, Brasil. http://www.ufmt.br \\ ${ }^{3}$ Autor para correspondência: Marliton Rocha Barreto,e-mail: mrbarreto@ufmt.br
}

BARRETO, M.R., MACHINER, R. \& SMIDERLE, E.C. Cerambycinae (Coleoptera, Cerambycidae) in Mato Grosso, Brazil. Biota Neotrop. 13(1): http://www.biotaneotropica.org.br/v13n1/en/abstract?inventory+ bn04213012013

Abstract: An inventory of Cerambycinae for the state of Mato Grosso, in particular the region of Sinop and Claudia, is presented. Thirty four species, distributed in 32 genera, are recorded for the state. Among them, four are new records for Mato Grosso: Anoplomerus rotundicollis G.-Méneville, 1844; Juiaparus mexicanus (Thomson, 1861); Poeciloxestia ochrotaenia (Bates, 1870); Thoracibidion ruficaudatum (Thomson, 1865).

Keywords: insecta, inventory, Matogrossense Amazon.

BARRETO, M.R., MACHINER, R. \& SMIDERLE, E.C. Cerambycinae (Coleoptera, Cerambycidae) em Mato Grosso, Brasil. Biota Neotrop. 13(1): http://www.biotaneotropica.org.br/v13n1/pt/abstract?inventory+ bn04213012013

Resumo: Um inventário dos Cerambycinae para o Estado de Mato Grosso, em especial a região de Sinop e Claudia, é apresentado neste trabalho. Trinta e quatro espécies, distribuídas em 32 gêneros, são registradas para o estado. Entre elas, quatro são novos registros para o Mato Grosso: Anoplomerus rotundicollis G.-Méneville, 1844; Juiaparus mexicanus (Thomson, 1861); Poeciloxestia ochrotaenia (Bates, 1870); Thoracibidion ruficaudatum (Thomson, 1865).

Palavras-chave: insecta, inventário, Amazônia Matogrossense.

*Esta é a publicação número 25 da série técnica do Núcleo de Estudos Biológicos da Amazônia Meridional - NEBAM/UFMT. 


\section{Introdução}

Nos trópicos, os coleópteros são abundantes e responsáveis por grandes prejuízos em espécies florestais, sobretudo insetos das famílias Curculionidae e Cerambycidae, pois estes desempenham papel importante na degradação da madeira (Gray 1972).

Antes do século XX, as maiores contribuições para o conhecimento das espécies da Amazônia brasileira foram, segundo Martins et al. (2006), realizadas por Adam White (Catalogue of the coleopterous insects in the collection of the British Museum) e Henry Walter Bates com a série (Contributions to an insect fauna of the Amazon Valley). Durante o século XX, um número muito grande de espécies foi descrita da região Amazônica, suplantando, e muito, aquelas descritas por Bates. Essas descrições iniciaram-se com Christopher Aurivillius, no inicio do século, com espécies do Peru, Bolívia e Brasil (região amazônica). Ao longo do século, vários autores acrescentarem espécies, bem como efetuaram revisões desses táxons. Rodrigues et al. (2006) relataram que o grupo é relativamente bem estudado, porém são escassos os trabalhos sobre a fauna de Cerambycidae para os principais biomas do país. Como exemplo desses trabalhos, podemos citar Maia et al. (2003), 66 espécies de Cerambycidae sendo 30 de Cerambycinae para a Caatinga; Galileo \& Martins (2006), para o Parque Copesul de Proteção Ambiental (RS); Monné et al. (2009a), 293 espécies de Cerambycinae para o Parque Nacional do Itatiaia (RJ); Monné et al. (2009b), 571 espécies para a região da Mata Atlântica do Estado do Rio de Janeiro; e Martins et al. (2009), 61 espécies para o cerrado maranhense e para a Região Amazônica, Souza \& Silva (2012) registraram 61 espécies com 33 novos registros para o estado de Rondônia.

Os Cerambycinae caracterizam-se pelas tíbias anteriores inteiramente roliças, desprovidas de sulco, pelo último segmento dos palpos frequentemente truncado no ápice, subtriangular, pela cabeça com fronte relativamente estreita e pouco inclinada em relação ao vértice, raramente vertical. Woldan (2007) relata que, de forma geral, Cerambycidae é reconhecida devido as suas longas antenas, embora alguns gêneros como, Parandra Latreille, 1802 e Hypocephalus Desmarest, 1832, tenham antenas bem mais curtas (Lima 1955). Essencialmente são fitófagos e com larvas xilófagas (Martins 1997). Algumas espécies apresentam considerável importância econômica por possuírem larvas broqueadoras de plantas cultivadas. No entanto, a subfamília também é importante no ciclo de nutrientes em seus habitats (Monné 2001). Devido à estreita relação com os recursos que utilizam, monofilias e especificidades quanto as relações ambientais e comportamentais, tornam-os candidatos a bioindicadores (Person 1994). Desta forma, Este trabalho visa fornecer subsídios para futuros estudos envolvendo a diversidade de Cerambycinae da região norte do Estado de Mato Grosso.

\section{Material e Métodos}

Os cerambicídeos listados neste trabalho foram coletados no período 2008-2010, em duas áreas florestais [Fazenda Continental ( $11^{\circ} 34^{\prime} 54.0^{\prime \prime} \mathrm{S}$ e $55^{\circ} 17^{\prime} 15.6$ " W), no município de Claudia e na Chácara Shalom ( $11^{\circ} 87^{\prime} 20.9^{\prime}$ ' S e $\left.55^{\circ} 47^{\prime} 48.6^{\prime \prime} \mathrm{W}\right)$, no município de Sinop]. As coletas foram realizadas no período noturno, com utilização de armadilha luminosa Tipo "Luiz de Queiroz" (lâmpada fluorescente de 20W).

No laboratório de entomologia da UFMT, Campus Universitário de Sinop, os insetos foram triados, montados e separados para identificação das espécies. Essa última etapa foi realizada pelo Dr. Miguel A. Monné do Museu Nacional, Universidade Federal do Rio de Janeiro, Rio de Janeiro (MNRJ) e o material estudado está depositado na coleção entomológica do Acervo Biológico da Amazônia Meridional da UFMT, sob números de registro ABAM - E', conforme apresentado no resultado.

A lista é apresentada em ordem alfabética de gênero e espécie e as referências bibliográficas para cada espécie seguem preferencialmente os catálogos e/ou checklist de Cerambycidae (Monné 2005, 2006, Monné \& Bezark 2011) embora outras tenha sido utilizadas. A distribuição geográfica de cada espécie segue as fontes mencionadas acima, bem como outras complementares e posteriormente adicionadas. Adotou-se o procedimento de indicar os países cuja ocorrência já tenha sido mencionada e, no caso do Brasil, quando possível, buscou-se apresentar os estados de ocorrência. Vale ressaltar que a presente lista não relaciona todas as espécies anteriormente citadas para o Estado, mas sim, apenas aquelas coletadas durante o estudo. Também, devido à divisão do Estado (Mato Grosso e Mato Grosso do Sul), citações antigas, nas quais não há uma localidade precisa, não podem ser confirmadas para o atual Estado do Mato Grosso.

\section{Resultados}

Do total de 64 insetos coletados, 32 espécies foram identificadas (59 exemplares) e apenas cinco insetos foram identificados a nível de gênero, 4 exemplares de Eburodacrys White, 1853 e 1 exemplar de Mionochroma Schmidt, 1924.

Registramos como nova ocorrência para a região de Sinop e Claudia, no Estado de Mato Grosso, as espécies: Anoplomerus rotundicollis G.-Méneville, 1844; Juiaparus mexicanus (Thomson, 1861); Poeciloxestia ochrotaenia (Bates, 1870); Thoracibidion ruficaudatum (Thomson, 1865).

Diversos fatores podem determinar a diversidade das espécies. Silveira (2010) relata que em uma escala mais ampla, os mecanismos mais influentes na adição e manutenção da diversidade de organismos associados são a área e a heterogeneidade do habitat. Da mesma forma, a variação quanto à diversidade de espécies pode ser atribuída às características próprias de cada local, em especial ao tipo de vegetação, sua conservação e quantidade e idade de material em decomposição. A diversidade de cerambicídeos é elevada em florestas secundárias. As possíveis causas da elevada riqueza de espécies em povoamentos humanos jovens incluem grandes quantidades de restos de madeira e flores, que são recursos para oviposição e alimentação para os adultos. A abertura do dossel e a variedade de plantas hospedeiras também influenciam a diversidade (Makino et al. 2007).

\section{CERAMBYCINAE}

\section{Achryson surinamum (Linnaeus, 1767)}

Distribuição: Estados Unidos, México, América Central, Porto Rico, América do Sul, Brasil (Rondônia, Goiás, Mato Grosso, Maranhão, Piauí, Alagoas, Sergipe, Bahia, Minas Gerais, Rio de Janeiro, Rio Grande do Sul).

Material examinado: BRASIL, Mato Grosso: Claudia, 21.VII.2010, (ABAM - E165).

\section{Acyphoderes abdominalis (Olivier, 1795)}

Distribuição: Nicarágua ao Panamá, Guiana, Suriname, Guiana Francesa, Equador, Peru, Brasil (Amazonas, Goiás, Mato Grosso), Bolívia.

Material examinado: BRASIL, Mato Grosso: Claudia, 09.X.2009, (ABAM - E187).

\section{Ambonus interrogationis (Blanchard, 1846)}

Distribuição: Brasil (Distrito Federal, Goiás, Mato Grosso, Mato Grosso do Sul, Piauí, Ceará, Rio Grande do Norte, Paraíba, Pernambuco, Bahia, Minas Gerais, Espírito Santo, Rio de Janeiro, São Paulo, Paraná, Santa Catarina, Rio Grande do Sul), Bolívia, Paraguai, Argentina. 
Material examinado: BRASIL, Mato Grosso: Sinop, 19.III.2010, (ABAM - E167).

\section{Anoplomerus rotundicollis G.-Méneville, 1844}

Distribuição: Brasil (Mato Grosso, Minas Gerais, Espírito Santo, Rio de Janeiro, São Paulo, Paraná, Santa Catarina), Paraguai, Argentina.

Material examinado: BRASIL, Mato Grosso: Sinop, 11.X.2008, (ABAM - E175).

\section{Batus barbicornis (Linnaeus, 1764)}

Distribuição: Venezuela, Guiana, Guiana Francesa, Colômbia, Equador, Peru, Brasil (Amapá, Amazonas, Pará, Rondônia, Goiás, Mato Grosso), Bolívia.

Material examinado: BRASIL, Mato Grosso: Sinop, 11.X.2008, (ABAM - E161).

\section{Ceragenia bicornis (Fabricius, 1801)}

Distribuição: Venezuela, Guiana, Guiana Francesa, Suriname, Equador, Peru, Brasil (Amazônia, Goiás, Mato Grosso, Maranhão), Bolívia.

Material examinado: BRASIL, Mato Grosso: Claudia, 11.X.2008, (ABAM - E180).

\section{Chlorida festiva (Linnaeus, 1758)}

Distribuição: Estados Unidos (Florida), América Central, Porto Rico, Brasil (Amazonas, Rondônia, Goiás, Mato Grosso, Maranhão, Piauí, Minas Gerais, Rio de Janeiro, São Paulo, Rio Grande do Sul), Argentina.

Material examinado: BRASIL, Mato Grosso: Claudia,19. III.2009, (ABAM - E183).

\section{Chydarteres dimidiatus dimidiatus (Fabricius, 1787)}

Distribuição: Colômbia, Guiana, Guiana Francesa, Brasil (Amazonas, Pará, Piauí, Pernambuco, Bahia, Goiás, Mato Grosso, Minas Gerais, Espírito Santo, Rio de Janeiro, São Paulo, Paraná, Santa Catarina, Rio Grande do Sul), Bolívia, Uruguai, Argentina. Material examinado: BRASIL, Mato Grosso: Sinop,11.X.2008, (ABAM - E162).

\section{Coccoderus longespinicornis Fuchs, 1964}

Distribuição: Venezuela, Guiana Francesa, Peru, Brasil (Amapá, Amazonas, Pará, Rondônia, Maranhão, Piauí, Distrito Federal, Goiás, Mato Grosso, Mato Grosso do Sul, Minas Gerais). Material examinado: BRASIL, Mato Grosso: Claudia, 27.X.2010, (ABAM - E163).

\section{Coleoxestia rubromaculata (Gounelle, 1909)}

Distribuição: Honduras, Nicarágua-Colômbia, Equador, Brasil (Rondônia, Goiás, Mato Grosso).

Material examinado: BRASIL, Mato Grosso: Claudia, 19.III.2010, (ABAM - E174).

\section{Dorcacerus barbatus (Olivier, 1790)}

Distribuição: México, Brasil (Amazonas, Pará, Goiás, Mato Grosso, Maranhão, Ceará, Rio Grande do Norte, Pernambuco, Alagoas, Sergipe, Minas Gerais, Rio de Janeiro, São Paulo, Paraná, Santa Catarina, Rio Grande do Sul), Argentina, Uruguai, Paraguai, Peru, Bolívia, Guiana Francesa.

Material examinado: BRASIL, Mato Grosso: Sinop, 23.V.2009, (ABAM - E171).

\section{Drychateres bilineatus (Olivier, 1795)}

Distribuição: Brasil (Rondônia, Goiás, Mato Grosso, Paraíba ao Rio Grande do Sul), Bolívia, Paraguai, Argentina, Uruguai.
Material examinado: BRASIL, Mato Grosso: Claudia, 16.X.2009, (ABAM - E182).

\section{Eburodacrys campestris Gounelle, 1909}

Distribuição: Brasil (Pará, Rondônia, Goiás, Mato Grosso, Maranhão, Piauí, Bahia a São Paulo), Paraguai, Argentina.

Material examinado: BRASIL, Mato Grosso: Sinop, 16.X.2009, (ABAM - E164).

\section{Eburodacrys sp.}

Material examinado: BRASIL, Mato Grosso: Sinop,11.X.2008, (ABAM - E178).

\section{Hexoplon uncinatum Gounelle, 1909}

Distribuição: Guiana Francesa, Peru, Bolívia, Brasil (Maranhão, Goiás, Mato Grosso, Mato Grosso do Sul, São Paulo), Paraguai, Argentina.

Material examinado: BRASIL, Mato Grosso: Claudia, 16.X.2009, (ABAM - E172).

\section{Juiaparus batus batus (Linnaeus, 1758)}

Distribuição: México ao Panamá, Venezuela, Colômbia, Guiana Francesa, Guiana, Suriname, Trinidad e Tobago Brasil (Amazonas, Amapá, Pará, Rondônia, Goiás, Mato Grosso, Maranhão, Ceará, Paraíba, Alagoas, Sergipe, Bahia).

Material examinado: BRASIL, Mato Grosso: Claudia, 23.V.2009, (ABAM - E189).

\section{Juiaparus mexicanus (Thomson, 1861)}

Distribuição: Colômbia, Guiana Francesa, Peru, México ao Brasil (Amazonas, Pará, Mato Grosso, Maranhão, Ceará, Paraíba, Pernambuco, Bahia, Minas Gerais, Espírito Santo, Rio de Janeiro, São Paulo, Paraná, Santa Catarina, Rio Grande do Sul), Bolívia.

Material examinado: BRASIL, Mato Grosso: Claudia,14. IX.2008, (ABAM - E185).

\section{Macrambyx suturalis (Gory, 1832)}

Distribuição: Guiana Francesa, Peru, Equador, Brasil (Amapá, Amazonas, Pará, Mato Grosso).

Material examinado: BRASIL, Mato Grosso: Sinop, 11.X.2008, (ABAM - E170).

\section{Malacopterus tenellus (Fabricius, 1801)}

Distribuição: Estados Unidos, México, América Central e Antilhas (Cuba, Guadalupe), Colômbia, Venezuela, Guiana, Suriname, Peru, Brasil(Amazonas, Pará, Mato Grosso), Bolívia, República Dominicana.

Material examinado: BRASIL, Mato Grosso: Claudia,11.X.2008, (ABAM - E173).

\section{Mallocera amazonica Bates, 1870}

Distribuição: Trinidad e Tobago, Guiana Francesa, Equador, Peru, Brasil (Amazonas, Pará, Rondônia, Maranhão, Goiás, Mato Grosso), Bolívia.

Material examinado: BRASIL, Mato Grosso: Claudia, 16.X.2009, (ABAM - E186).

\section{Mionochroma sp.}

Material examinado: BRASIL, Mato Grosso: Sinop, 11.X.2008, (ABAM - E197).

\section{Neomegaderus stigma (Linnaeus, 1758)}

Distribuição: Nicarágua, Bolívia, Brasil (Goiás, Mato Grosso, Maranhão, Ceará, Paraíba, Pernambuco, Bahia, Minas Gerais, Espírito Santo, Rio de Janeiro, São Paulo), Argentina, Uruguai, 
Guiana Francesa, Costa Rica, Antilhas, Paraguai, Panamá, Colômbia, Peru, Panamá, Suriname.

Material examinado: BRASIL, Mato Grosso: Claudia, 27.X.2010, (ABAM - E166)

\section{Neotropidion nodicolle nodicolle (Dalman, 1823)}

Distribuição: Venezuela, Guiana Francesa, Equador, Peru, Brasil (Amazonas, Acre, Pará, Rondônia, Mato Grosso, Bahia a São Paulo), Bolívia.

Material examinado: BRASIL, Mato Grosso: Claudia, 14.IX.2008, (ABAM - E169).

\section{Nyssicus quadriguttatus (Swederus, 1787)}

Distribuição: Guiana Francesa, Equador, Peru, Brasil (Amazonas, Pará, Rondônia, Mato Grosso).

Material examinado: BRASIL, Mato Grosso: Sinop, 11.X.2008, (ABAM - E201).

\section{Oxymerus basalis (Dalman, 1823)}

Distribuição: Martinica, Venezuela, Guiana Francesa, Peru, Brasil (Amazonas, Pará, Rondônia, Goiás, Mato Grosso, Maranhão, Ceará a São Paulo),

Material examinado: BRASIL, Mato Grosso: Sinop, 23.V.2009, (ABAM - E192).

\section{Paranyssicus conspicillatus (Erichson, 1847)}

Distribuição: Guiana Francesa, Peru, Brasil (Amazonas, Pará, Rondônia, Maranhão, Mato Grosso, Goiás), Bolívia, Equador.

Material examinado: BRASIL, Mato Grosso: Sinop, 16.X.2009, (ABAM - E179).

\section{Poeciloxestia ochrotaenia (Bates, 1870)}

Distribuição: Brasil (Amazonas, Pará, Mato Grosso). Material examinado: BRASIL, Mato Grosso: Sinop, 23.V.2009, (ABAM - E176).

\section{Sphallotrichus puncticollis puncticollis (Bates, 1870)}

Distribuição: Guiana Francesa, Brasil (Amapá, Amazonas, Pará, Mato Grosso).

Material examinado: BRASIL, Mato Grosso: Sinop, 19.III.2010, (ABAM - E168).

\section{Susuacanga unicolor (Bates, 1870)}

Distribuição: Venezuela, Colômbia, Brasil (Acre, Amazonas, Pará, Rondônia, Ceará, Goiás, Mato Grosso, Minas Gerais, Rio de Janeiro, São Paulo,), Peru, Bolívia, Paraguai.

Material examinado: BRASIL, Mato Grosso: Claudia, 23.V.2009, (ABAM - E177).

\section{Tapuruia felisbertoi Lane, 1973}

Distribuição: Guiana Francesa, Brasil (Amazonas, Pará, Goiás, Mato Grosso), Bolívia.

Material examinado: BRASIL, Mato Grosso: Sinop, 23.V.2009, (ABAM - E181).

\section{Thoracibidion ruficaudatum (Thomson, 1865)}

Distribuição: Venezuela, Guiana Francesa, Brasil (Amapá, Amazonas, Pará, Rondônia, Mato Grosso), Peru.

Material examinado: BRASIL, Mato Grosso: Sinop, 14.IX.2008, (ABAM - E195).

\section{Tobipuranga ruficoxis (Bates, 1870)}

Distribuição: Equador, Peru, Brasil (Amazonas, Pará, Rondônia, Mato Grosso), Bolívia.
Material examinado: BRASIL, Mato Grosso: Sinop, 11.X.2008, (ABAM - E207).

\section{Trachyderes succinctus succinctus (Linnaeus, 1758)}

Distribuição: Guadalupe, Costa Rica, Barbados, Nicarágua, Honduras, Argentina, México, Costa Rica, Panamá, Pequenas Antilhas (São Martin, Guadalupe, Martinica), Trinidad e Tobago, Grandes Antilhas (São Tomás, Ilhas Virgens), Colômbia, Venezuela, Suriname, Guiana, Brasil (Amapá, Pará, Mato Grosso, Maranhão, Piauí, Ceará, Paraíba, Pernambuco, Bahia, Minas Gerais, Espírito Santo, Rio de Janeiro, São Paulo, Rio Grande do Sul), Equador, Peru, Bolívia.

Material examinado: BRASIL, Mato Grosso: Claudia, 11.X.2008, (ABAM - E215).

\section{Tropidion subcruciatum (White, 1855)}

Distribuição: Venezuela, Suriname, Guiana Francesa, Colômbia, Brasil (Amazônia, Goiás, Mato Grosso, Mato Grosso do Sul, Maranhão, Paraíba ao Paraná), Bolívia.

Material examinado: BRASIL, Mato Grosso: Sinop. 14.IX.2008, (ABAM - E198).

\section{Agradecimentos}

Ao Dr. Miguel A. Monné do Museu Nacional da Universidade Federal do Rio de Janeiro pela identificação dos insetos. Ao CNPq (Processo No 569382/2008-4) e a FAPEMAT (processos $n^{\circ}$ $688844 / 2010$ e $300729 / 2010$ ), pelo auxílio financeiro.

\section{Referências Bibliográficas}

GALILEO, M.H.M. \& MARTINS, U.R. 2006. Cerambycidae (Coleoptera) - Parque Copesul de Proteção Ambiental, Triunfo, Rio Grande do Sul. Museu de Ciências Naturais da Fundação Zoobotânica do Rio Grande do Sul, Porto Alegre, p.1-316.

GRAY, B. 1972. Economic tropical forest entomology. Ann. Rev. Entomol. 17:313-354. http://dx.doi.org/10.1146/annurev. en.17.010172.001525

LIMA, A.M.C. 1955. Insetos do Brasil, Coleópteros. Escola Nacional de Agronomia, Rio de Janeiro, v.9, p.1-138.

MAIA, A.C.D., IANNUZZI, L., NOBRE, C.E.B. \& ALBUQUERQUE, C.M. 2003. Padrões locais de diversidade de Cerambycidae (Insecta, Coleoptera) em vegetação de caatinga. In: Ecologia e Conservação da Caatinga (I.R. Leal, M. Tabarelli \& J.M.C. Silva, orgs.). Editora Universitária da Universidade Federal de Pernambuco, Recife, p.391-433.

MAKINO, S., GOTO, H., HASEGAWA, M., KIMIKO OKABE, K., TANAKA, H., INOUE, T. \& OKOCHI, I. 2007. Degradation of longicorn beetle (Coleoptera, Cerambycidae, Disteniidae) fauna caused by conversion from broad-leaved to man-made conifer stands of Cryopomeria japonica (Taxodiaceae) in central Japan. Eco. Res. 22(3):134-156. http://dx.doi. org/10.1007/s11284-007-0359-y

MARTINS, U.R. 1997. Cerambycidae Sul-Americanos. Sociedade Brasileira de Entomologia, São Paulo, v.1, p.1-217.

MARTINS, U.R., GALILEO, M.H.M. \& LIMEIRA-DE-OLIVEIRA, F. 2009. Cerambycidae (Coleoptera) do estado do Maranhão, Brasil. Pap. Avulsos Zoo. 49(19):229-247.

MARTINS, U.R.; GALILEO, M.H.M.; SANTOS-SILVA, A. \& RAFAEL, J.A. 2006. Cerambycidae (Coleoptera) coletados à luz a 45 metros de altura, no dossel da floresta amazônica, e a descrição de quatro espécies novas. Acta Amazon. 36(2):265-272. http://dx.doi.org/10.1590/S004459672006000200017

MONNÉ, M.A. \& BEZARK, L. 2011. Checklist of the Cerambycidae, or longhorned beetles (Coleoptera) of the western Hemisphere. http://plant. cdfa.ca.gov./byciddb/documents.html (último acesso em 05/06/2011).

MONNÉ, M.A. 2005. Catalogue of the Cerambycidae (Coleoptera) of the Neotropical Region. Part I. Subfamily Cerambycinae. Zootaxa 946:1-765. 
MONNÉ, M.A. 2001. Catalogue of Neotropical Cerambycidae (Coleoptera) with known host plant - part I, Subfamily Cerambycinae, tribes Acrysonini to Elaphidiini. Publ. avulsas do Mus Nac. 88:1-108.

MONNÉ, M.L., MONNÉ, M.A. \& MERMUDES, J.R.M. 2009a. Inventário das espécies de Cerambycinae (Insecta, Coleoptera, Cerambycidae) do Parque Nacional do Itatiaia, Estado do Rio de Janeiro, Brasil. Biota neotrop. 9(3):283-312.

MONNÉ, M.L., MONNÉ, M.A., MARTINS, R.S., SIMÕES, M.V.P. \& MACHADO, V.S. 2009b. Espécies de Cerambycidae (Insecta, Coleoptera) ocorrentes no Estado do Rio de Janeiro (Brasil). Arq. Mus. Nac. 67(3-4):235-251.

PERSON, D.L. 1994. Selecting indicator taxa for the quantitative assessment of biodiversity. Philos. T. Roy. Soc. B. 345:75-79. http://dx.doi. org/10.1098/rstb.1994.0088
RODRIGUES, J.M.S., MONNÉ, M.A. \& MERMUDES, J.R.M. 2006. Inventário das espécies de Cerambycidae (Coleoptera) de Vila Dois Rios (Ilha Grande, Angra dos Reis, Rio de Janeiro, Brasil). Biota neotrop. 10(3):311-321. http://dx.doi.org/10.1590/S167606032010000300029

SILVEIRA, M.A.P.A. 2010. Analise faunística de insetos nas margens do alto rio Madeira, Porto Velho, Rondônia. Brasil. Tese de Doutorado, Escola Superior de Agricultura Luiz de Queiroz da Universidade de São Paulo, São Paulo. 73 p.

SOUZA, D.S. \& SILVA, A.A.. 2012. Cerambycidae (Insecta: Coleoptera) of the Parque Natural Municipal de Porto Velho, Rondônia, Western Amazon, Brazil. Biota Neotrop. 12(1):237-240. http://dx.doi.org/10.1590/ S1676-06032012000100018

WOLDAN, D.R.H. 2007. Análise faunística de Cerambycidae (Coleoptera) em duas situações florísticas no município de União da Vitória - Paraná. Dissertação de Mestrado, Universidade Comunitária Regional de Chapecó.

Recebido em 21/11/2012 Versão reformulada recebida em 25/02/2013 Publicado em 29/03/2013 
